\title{
Impact of whole grains on the gut microbiota: the next frontier for oats?
}

\author{
Devin J. Rose* \\ Department of Food Science and Technology, University of Nebraska-Lincoln, 143 Filley Hall, Lincoln, NE 68583, USA
}

(Submitted 7 October 2013 - Final revision received 18 March 2014 - Accepted 6 May 2014)

\begin{abstract}
The gut microbiota plays important roles in proper gut function and can contribute to or help prevent disease. Whole grains, including oats, constitute important sources of nutrients for the gut microbiota and contribute to a healthy gut microbiome. In particular, whole grains provide NSP and resistant starch, unsaturated TAG and complex lipids, and phenolics. The composition of these constituents is unique in oats compared with other whole grains. Therefore, oats may contribute distinctive effects on gut health relative to other grains. Studies designed to determine these effects may uncover new human-health benefits of oat consumption.
\end{abstract}

Key words: Bacteria: Prebiotic: Health: SCFA

The role that human colonic bacteria, or the gut microbiota, plays in health and disease is an area of research with intense current interest that is fuelled by provocative reports suggesting that the gut microbiota plays a role in many diseases that plague the modern society, including obesity, diabetes and colorectal cancer ${ }^{(1)}$. Diet plays an important, albeit incompletely understood, role in maintaining healthy gut-microbiota interactions ${ }^{(2)}$. While a wealth of research has focused on prebiotics and isolated dietary fibres and their contribution to gut health ${ }^{(3)}$, little research has been conducted on how dietary fibres and other constituents from whole-food matrices affect the gut microbiota.

Whole grains, for instance, are rich sources of dietary fibres and other bioactive compounds ${ }^{(4,5)}$ that may modulate the gut microbiota and thereby impact on consumer health ${ }^{(6,7)}$. In particular, oats may contribute specialised influences on the gut microbiota in comparison with other grains. Although most cereals contain very little soluble dietary fibre, oats contain high levels of soluble fibre in the form of mixed linkage $(1 \rightarrow 3),(1 \rightarrow 4)$ - $\beta$-D-glucan

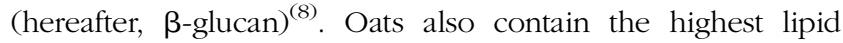
content among the major grains together with potential bioactives ${ }^{(9,10)}$. This article discusses the effects of whole grains on gut health and then highlights some of the unique aspects of oats that may contribute to enhanced gut health.

\section{Influence of whole grains on the gut microbiota}

Although the principal non-digestible components in whole grains are cross-linked arabinoxylan and cellulose (see online supplementary Table S1) - substrates that are generally considered poor for gut microbial fermentation - whole grains have the potential to play an important role in maintaining a healthy gut microbiota. Two human trials have suggested a bifidogenic effect from the consumption of whole-grain cereals $^{(6,7)}$. Some strains of Bifidobacterium have been reported in the literature as markers of a healthy gut microbiota ${ }^{(3)}$. Other studies have shown increases in butyrate-producing bacteria, including Roseburia, Eubacterium rectale and the Clostridium leptum group (which includes Faecalibacterium

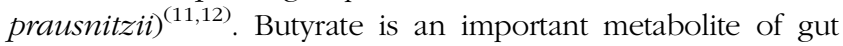
microbial fermentation of carbohydrates for its contribution to host colonic epithelial cell energy, anti-inflammatory properties and anti-cancer effects ${ }^{(13)}$. Whole grains have been shown to decrease protein fermentation by the gut microbiota ${ }^{(14)}$. Protein fermentation is associated with undesirable fermentation metabolites and detrimental effects on the host ${ }^{(15)}$. Whole grains may also increase the diversity of the microbiota ${ }^{(12)}$, a potential benefit considering that reduced diversity is associated with obesity and disease ${ }^{(16,17)}$. Notably, consumption of many purified dietary fibres has not been shown to increase diversity ${ }^{(18,19)}$.

The beneficial effects of whole grains on health are likely to be a combined result of many components within the grain rather than one specific component ${ }^{(5)}$. Three components of whole grains that are likely to impact the gut microbiota with trophic effects on the host are discussed later.

\section{Dietary fibre}

The insoluble, NSP in cereals have long been recognised for their faecal bulking properties and reduction in gastrointestinal

Abbreviation: RS, resistant starch.

*Corresponding author: D. J. Rose, email drose3@unl.edu 
transit time ${ }^{(20)}$. These effects may be particularly important for older adults, who typically consume less dietary fibre and have reduced gastric motility ${ }^{(21)}$. However, because of their poor utilisation by colonic bacteria, the impact of NSP on the gut microbiota is often overlooked. The poorly fermentable, dietary fibres in whole grains contribute to reduced distal colonic $\mathrm{pH}$ and higher faecal butyrate concentrations ${ }^{(22,23)}$. This is likely because the poor fermentation of the NSP allows it to persist into the distal colon and provide some carbohydrate substrate for gut bacterial metabolism in this region, a characteristic not attributable to most soluble and highly fermentable dietary fibres. Such conditions are consistent with reduced inflammation and carcinoma development ${ }^{(13,24)}$.

In addition to the principal NSP in whole grains, they also contain water-extractable arabinoxylan and mixed-linkage $\beta$-glucan (see online supplementary Table S1). The extent of fermentation and the types of bacteria that are favoured with these polymers depend on solubility, monosaccharide composition, presence of non-carbohydrate moieties, molecular weight and glycosidic linkages ${ }^{(25,26)}$. Viscosity was reported to affect the types of bacterial groups that are enriched with $\beta$-glucan ${ }^{(27)}$, although because the viscous property of $\beta$-glucan is rapidly lost upon contact with gut bacteria ${ }^{(28)}$ it is likely that these differences were due to molecular weight rather than viscosity. Fermentation of isolated water-extractable arabinoxylan and $\beta$-glucan by human intestinal bacteria in vitro is generally associated with an enrichment in propionate production compared with other dietary fibres ${ }^{(25,29-34)}$. Important propionate producers in the gut microbiota are members of clostridial cluster IX and Bacteroides ${ }^{(35)}$. Neither one of these polysaccharides is particularly bifidogenic, although bifidogenicity increases when partially hydrolysed ${ }^{(25,36)}$.

Whole grains also contain widely varying quantities of resistant starch (RS), depending on the types of grain used and product and processing conditions ${ }^{(37)}$. RS has received considerable attention in recent years for its influence on the gut microbiota and subsequent impacts on the host ${ }^{(18)}$. Even though the exact content type of RS in most whole-grain foods is fairly modest (typically $1-3 \%)^{(37)}$, this may still have a positive impact on health. For instance, in subjects who consumed whole-wheat or wheat-bran cereals, a bifidogenic effect was only observed in the whole-wheat cereal ${ }^{(6)}$. The difference between the two cereals was mainly the starch content. This suggests that the prebiotic effect of the whole-grain cereals could have been at least partially a result of RS fermentation, as RS has shown prebiotic properties $^{(18)}$. Furthermore, RS is also particularly butyrogenic, and in an in vitro trial, Costabile et al. ${ }^{(6)}$ found greater butyrate production when the whole-grain wheat cereal was fermented with faecal bacteria compared with the wheat-bran cereal.

Finally, whole grains contain about $0 \cdot 1-1 \% \alpha$-galactosyl derivatives of sucrose (mainly raffinose and stachyose) and $0 \cdot 3-4 \% \beta$-fructosyl derivatives of sucrose (referred to as fructan; see online supplementary Table S1). These carbohydrates are generally located in the outer portions of the grain, with raffinose and stachyose concentrated in the germ ${ }^{(5)}$. Although their content in grain products is fairly low compared with some other plants, the prevalence of grain-based foods in the diet makes them an important dietary source of these carbohydrates $^{(38)}$. Raffinose and stachyose are rapidly fermentable and bifidogenic ${ }^{(3)}$. These carbohydrates also possess several health benefits ${ }^{(39)}$. When consumed as isolated ingredients, they can cause boating, flatulence and, at very high intakes, diarrhoea ${ }^{(39)}$; however, these high intakes are not likely to be achieved through whole-grain consumption. The fructans in whole grains contain an average degree of polymerisation of four to six with few oligomers containing more than nine degrees of polymerisation ${ }^{(40)}$. Furthermore, fructans in whole grains contain branched structures with both $(2 \rightarrow 1)$ and $(2 \rightarrow 6) \beta$-fructosyl linkages ${ }^{(41)}$. No studies have isolated fructans from any whole grain and determined its fermentation properties; however, short-chain fructan oligomers are generally rapidly fermented by gut bacteria and highly bifidogenic ${ }^{(3)}$. Several reviews have demonstrated the positive impact that fructans impart on human health ${ }^{(42,43)}$

\section{Lipids}

Evidence in mice has suggested that diets high in fat in the form of bulk lipids (predominantly TAG) have a detrimental effect on the gut microbiota and host metabolic parameters ${ }^{(44)}$ In particular, diets high in saturated fats have been shown to decrease proportions of beneficial bacteria ${ }^{(45)}$ and decrease microbial diversity ${ }^{(46)}$. de Wit et al. ${ }^{(46)}$ suggested that saturated fats were especially detrimental to the gut microbiota because they persisted into the distal small intestine where they exhibited an antimicrobial effect (and consequently reduced diversity). Whole grains are comparatively low in fat, and the fat that they do contain is highly unsaturated.

Whole-grain lipids themselves may also more directly affect the gut microbiota with subsequent influences on health. For instance, hamsters fed diets supplemented with up to $5 \%$ wholegrain sorghum lipids showed significantly increased Bifidobacteria and decreased Coriobacteriaceae in the faeces $^{(47)}$. Bifidobacteria concentration was positively correlated with plasma HDL-concentration, while Coriobacteriaceae were positively correlated with non-HDL-cholesterol and cholesterol absorption. In another study ${ }^{(48)}$, rice bran oil incorporated into the diets of mice increased bile acids in the faeces with accompanying positive correlations with the family Lactobacillales (which contains lactic acid bacteria) Martínez et al. ${ }^{(49)}$ theorised that this was due to the sterol esters in the lipid extract (about 10\%). When hamsters were fed purified steryl esters, cholesterol absorption decreased, which led to an increase in the cholesterol pool in the gut and a decrease in Coriobacteriaceae and Erysipelotrichaceae. These two families have shown positive correlations with deleterious host lipid parameters ${ }^{(47,50)}$. Whole grains are rich sources of plant sterol esters in comparison with other foods ${ }^{(51)}$. In addition, other lipid components of grains, for example the policosanols, may also affect the gut microbiota, although this remains to be determined.

\section{Phenolics}

The majority of the antioxidants in whole grains are bound to dietary fibre components, precluding their absorption in the 
upper gastrointestinal tract ${ }^{(52)}$. However, microbial-derived esterases in the lower gastrointestinal tract can release a portion of these compounds from their dietary fibre conjugates, whereupon they are rapidly metabolised. Ferulic acid, the major phenolic acid in whole grains, for instance, is first hydrogenated at the double bond on the propen-2-oic acid side chain followed by demethylation of the ring diethyl ether. Subsequently, this metabolite, 3,4-dihydroxyphenylpropionic acid, is dehydroxylated to 3-hydroxyphenylpropionic acid and phenylpropionic acid ${ }^{(53)}$. Dimers and oligomers of ferulic acid are also present in whole grains and metabolised either by direct modification of the functional groups or by deconjugation and metabolism as monomers ${ }^{(54)}$. These metabolites may impart beneficial biological activity ${ }^{(55)}$, although there are still many questions regarding the effective dose, bioavailability and pharmacokinetics of these compounds in humans.

While microbial esterases are effective at removing a variable portion of bound phenolics, which depends on many factors ${ }^{(53)}$, many phenolics are still not biologically available $^{(56)}$. However, the physical presence of high concentrations of bound phenolic antioxidants in the gastrointestinal tract may itself impart several benefits. While the body contains natural defence mechanisms against free radical oxidation, the lumen of the gastrointestinal tract is not protected to the same extent. Furthermore, under some circumstances, such as during inflammation, neutrophils produce free radicals (e.g. $\mathrm{HNO}_{3}$ ) that can lead to mucosal oxidative stress, peroxidation of membrane lipids and tissue damage ${ }^{(57,58)}$. The presence of bound antioxidants in the lumen, such as those in whole grains, may protect the intestinal epithelium from free radical damage ${ }^{(56)}$. This is supported by studies showing that the physical presence of ferulic acid in the lumen of the large intestine, administered via enema, leads to reduced inflammation in inflammatory bowel disease patients ${ }^{(59)}$.

\section{What about oats?}

As with other whole grains, in vivo trials on the effects of oats on markers of gut health are sparse (see online supplementary Table S2). There are comparatively more in vitro studies; however, the data are far from comprehensive and in many cases conflicting (see online supplementary Table S3). Nevertheless, as mentioned, oats contain a unique chemical composition compared with other grains. The most prevalent differences are in the dietary-fibre composition, the lipid content and the types of phenolics. Because these three fundamental components are likely to have strong effects on the gut microbiota, as discussed, oats are likely to impact the gut microbiota in unique ways compared with other cereal grains. Studies aimed at elucidating these effects may help researchers further establish the mechanisms behind the health benefits of oats, and help breeders and food companies create oats and oat products that maximise the health benefits derived from this grain.

\section{Dietary fibre}

The high content of $\beta$-glucan is the most unique dietary fibre component in oats. While the viscous property of $\beta$-glucan is rapidly diminished once $\beta$-glucan is exposed to microbial metabolism in the large bowel ${ }^{(28)}$, fermentation of $\beta$-glucan by some gut microbes may play a role in the effects of whole-grain oats and barley on heart diseases. For instance, as mentioned, fermentation of isolated $\beta$-glucan generally enhances the production propionate by the gut microbiota $^{(25,31,33)}$, and studies suggest that propionate may help reduce serum cholesterol ${ }^{(60)}$. $\beta$-Glucan may also contribute to reduced colonic $\mathrm{pH}$ compared with other cereals containing less soluble (and fermentable) dietary fibres ${ }^{(61)}$.

RS content of oats may be an important contributor to its effects on gut health. Connolly et al. ${ }^{(62)}$ studied the differences in in vitro fermentation properties between thin and thick oat flakes (rolled oats). The thick oat flakes resulted in a significant increase in Bifidobacterium during fermentation compared with the baseline; this was not observed in the thin oat flakes. Moreover, the thick oat flakes resulted in 2.5 times more butyrate than the thin oat flakes. The authors suggested that the bifidogenic effect and the enhanced butyrate production in the presence of thick oat flakes were results of higher RS in these samples compared with the thin oat flakes. This was supported by another study where oat flours were subjected to in vitro digestion to remove digestible components before faecal fermentation; however, the residue still contained about $15 \% \operatorname{starch}^{(63)}$. The subsequent fermentation resulted in an acetate-propionate-butyrate ratio of about $42: 24: 35$, which is typical of starch-containing preparations ${ }^{(64)}$. High $\beta$-glucan in oat and barley cereals has been shown to reduce starch digestion in the small intestine due to its viscosity ${ }^{(65)}$. Thus, it is possible that oats and barley products contain higher RS than products from other grains because the starch digestion rate may be sufficiently slow as to allow a greater portion of starch to remain intact and reach the colon ${ }^{(66)}$.

In spite of their similarities in dietary fibre composition, oats and barley seem to induce different effects on the gut microbiota. For example, pigs consuming an oat-based diet showed significantly greater increases in bifidobacteria compared with pigs consuming a barley-based diet ${ }^{(67)}$. Another study by the same group showed a greater bifidogenic effect on an oat-based diet compared with a wheat-based diet that was supplemented with $\beta$-glucan such that the two diets had a similar $\beta$-glucan content ${ }^{(68)}$. The different influences that oats and barley have on the gut microbiota may be due to differences in the structures and molecular weight of $\beta$-glucan or other polysaccharides, as well as differences in non-dietary fibre components.

\section{Lipids}

Although lipid components from whole grains affect the gut microbiota, more research is needed to describe such effects in detail. No studies have demonstrated the effects of isolated oat lipids on the gut microbiota. In the studies cited earlier, plant sterols may affect changes in the gut microbiota with positive impacts on health. Oats contain moderate concentrations of sterols $(329-520 \mathrm{mg} / \mathrm{kg})$ : lower than wheat and rye (603-715 and $910-1100 \mathrm{mg} / \mathrm{kg}$, respectively), but higher than rice and sorghum (216-401 and 200-350 mg/kg, respectively) ${ }^{(51,69,70)}$. 


\section{Phenolics}

While the majority of phenolics in oats are bound ferulic acid, which is similar to other whole grains, oats also contain some unique phenolics including avenacosylates, avenacins and avenanthramides. Avenacosylates are esters of ferulic or caffeic acid with a long-chain wax alcohol (policosanol) and are present in oats at about 50-200 $\mathrm{mg}$ ferulic acid equivalents $/ \mathrm{kg}^{(9)}$. The avenacins are pentacyclic triterpene alcohol glycosides containing esters of aminophenolic or benzoic acid ${ }^{(9)}$. Avenanthramides are conjugates of a phenylpropanoid with anthranilic acid or 5-hydroxy anthranilic acid and are present in oats in widely varying concentrations similar to the avenocosylates ${ }^{(9)}$. It is likely that these compounds are metabolised by the gut microbiota, but this has not been reported in detail ${ }^{(71)}$. Some data suggest that they may function as anti-inflammatory molecules and protect the gut mucosa by modulating NF- $\mathrm{KB}$ activation ${ }^{(72)}$.

\section{Conclusions}

Whole-grain dietary fibres, despite being poorly fermented in many cases, are the major contributors to distal colonic fermentation $^{(22,23)}$, which results in a decrease in $\mathrm{pH}$ and potentially reduces the risk of some gastrointestinal diseases $^{(73)}$. Furthermore, RS may be a significant contributor to the benefits of whole grains ${ }^{(66)}$ and could be responsible for the bifidogenic effect of whole-grain cereals previously reported $^{(6,7)}$. Other constituents in whole grains that are often ignored in relation to gut health, namely the lipids and phenolic constituents, may also contribute to gut health ${ }^{(4,5)}$.

Oats are a unique whole grain that may contribute distinctive effects on the gut microbiota. These effects could be due to the high $\beta$-glucan content, the high lipid content or the unique antioxidant profile. $\beta$-Glucan fermentation could contribute to the hypocholesterolaemic properties of oats $^{(25)}$. Furthermore, $\beta$-glucan may slow the rate of starch digestion and help increase the RS content of oats relative to other grains that are low in $\beta$-glucan ${ }^{(65,66)}$. Oat lipids and antioxidants have not been studied in relation to gut health, but research in other whole grains suggests that they influence the types of bacteria that make up the microbiota and impact on host health ${ }^{(47,55,72)}$. The microbiota and gut health are at the intersection of emerging research, particularly when considering the demonstrated and possible health implications of whole grains and dietary fibre that are identified in oats.

\section{Supplementary material}

To view supplementary material for this article, please visit http://dx.doi.org/10.1017/S0007114514002244

\section{Acknowledgements}

D. J. R. received an honorarium from Quaker Oats Company (a subsidiary of PepsiCo, Inc.) for attending a workshop in May 2012 to discuss the content of the supplement, and University of Nebraska received an unrestricted grant from Quaker Oats Company.
This paper was published as part of a supplement to British Journal of Nutrition, publication of which was supported by an unrestricted educational grant from Quaker Oats Co. (a subsidiary of PepsiCo Inc.). The papers included in this supplement were invited by the Guest Editor and have undergone the standard journal formal review process. They may be cited.

The Guest Editor to this supplement is Roger Clemens. The Guest Editor declares no conflict of interest.

\section{References}

1. de Vos WM \& de Vos EA (2012) Role of the intestinal microbiome in health and disease: from correlation to causation. Nutr Rev 70, S45-S56.

2. Delzenne NM \& Cani PD (2011) Interaction between obesity and the gut microbiota: relevance in nutrition. Annu Rev Nutr 31, 15-31.

3. Gibson GR, Probert HM, Van Loo J, et al. (2004) Dietary modulation of the human colonic microbiota: updating the concept of prebiotics. Nutr Res Rev 17, 259-275.

4. Slavin JL (2003) Why whole grains are protective: biological mechanisms. Proc Nutr Soc 62, 129-134.

5. Fardet A (2010) New hypotheses for the health-protective mechanisms of whole-grain cereals: what is beyond fibre? Nutr Res Rev 23, 65-134.

6. Costabile A, Klinder A, Fava F, et al. (2008) Whole-grain wheat breakfast cereal has a prebiotic effect on the human gut microbiota: a double-blind, placebo-controlled, crossover study. Br J Nutr 99, 110-120.

7. Carvalho-Wells AL, Helmolz K, Nodet C, et al. (2010) Determination of the in vivo prebiotic potential of a maize-based whole grain breakfast cereal: a human feeding study. $\mathrm{Br} J$ Nutr 104, 1353-1356.

8. Ward JL, Poutanen K, Gebruers K, et al. (2008) The HEALTHGRAIN Cereal Diversity Screen: concept, results, and prospects. J Agric Food Chem 56, 9699-9709.

9. Collins FW (2011) Oat phenolics: biochemistry and biological functionality. In Oats: Chemistry and Technology, 2nd ed., pp. 157-218 [FH Webster and PJ Wood, editors] St Paul, MN: American Association of Cereal Chemists.

10. Lehtinen P \& Kaukovirta-Norja A (2011) Oat lipids, enzymes, and quality. In Oats: Chemistry and Technology, 2nd ed., pp. 143-156 [FH Webster and PJ Wood, editors]. St Paul, MN: American Association of Cereal Chemists.

11. Ross AB, Bruce SJ, Blondel-Lubrano A, et al. (2011) A wholegrain cereal-rich diet increases plasma betaine, and tends to decrease total and LDL-cholesterol compared with a refinedgrain diet in healthy subjects. Br J Nutr 105, 1492-1502.

12. Martínez I, Lattimer JM, Hubach KL, et al. (2013) Gut microbiome composition is linked to whole grain-induced immunological improvements. ISME J 7, 269-280.

13. Hamer HM, Jonkers D, Venema K, et al. (2008) Review Article: the role of butyrate on colonic function. Aliment Pharmacol Ther 27, 104-119.

14. Ross AB, Pere-Trépat E, Montoliu I, et al. (2013) A wholegrain-rich diet reduces urinary excretion of markers of protein catabolism and gut microbiota metabolism in healthy men after one week. J Nutr 143, 766-773.

15. Russell WR, Gratz SW, Duncan SH, et al. (2011) Highprotein, reduced-carbohydrate weight-loss diets promote metabolite profiles likely to be detrimental to colonic health. Am J Clin Nutr 93, 1062-1072. 
16. Turnbaugh PJ, Hamady M, Yatsunenko T, et al. (2009) A core gut microbiome in obese and lean twins. Nature $\mathbf{4 5 7}$, 480-484

17. Sha SM, Xu B, Wang X, et al. (2013) The biodiversity and composition of the dominant fecal microbiota in patients with inflammatory bowel disease. Diagn Microbiol Infect Dis 75, 245-251.

18. Martinez I, Kim J, Duffy PR, et al. (2010) Resistant starches types 2 and 4 have differential effects on the composition of the fecal microbiota in human subjects. PLOS ONE $\mathbf{5}$, e15046.

19. Davis LM, Martinez I, Walter J, et al. (2011) Barcoded pyrosequencing reveals that consumption of galactooligosaccharides results in a highly specific bifidogenic response in humans. PLOS ONE 6, e25200.

20. Graditske HA \& Slavin JL (2008) Laxation and the like: assessing digestive health. Nutr Today 43, 193-198.

21. Bhutto A \& Morley JE (2008) The clinical significance of gastrointestinal changes with aging. Curr Opin Clin Nutr Metab Care 11, 651-660.

22. McIntosh GH, Noakes M, Royle PJ, et al. (2003) Whole-grain rye and wheat foods and markers of bowel health in overweight middle-aged men. Am J Clin Nutr 77, 967-974.

23. Bird AR, Flory C, Davies DA, et al. (2004) A novel barley cultivar (Himalaya 292) with a specific gene mutation in starch synthase IIa raises large bowel starch and short-chain fatty acids in rats. $J$ Nutr 134, 831-835.

24. Medina V, Afonso JJ, Alvarez-Arguelles H, et al. (1998) Sodium butyrate inhibits carcinoma development in a 1,2dimethylhydrazine-induced rat colon cancer. JPENJ Parenter Enteral Nutr 22, 14-17.

25. Hughes SA, Shewry PR, Gibson GR, et al. (2008) In vitro fermentation of oat and barley derived $\beta$-glucans by human faecal microbiota. FEMS Microbiol Ecol 64, 482-493.

26. Pollet $\mathrm{A}$, Van Craeyveld $\mathrm{V}$, Van de Wiele $\mathrm{T}$, et al. (2012) In vitro fermentation of arabinoxylan oligosaccharides and low molecular mass arabinoxylans with different structural properties from wheat (Triticum aestivum $\mathrm{L}$.) bran and psyllium (Plantago ovata Forsk) seed husk. J Agric Food Chem 60, 946-954.

27. Metzler-Zebeli BU, Hooda S, Pieper R, et al. (2010) Nonstarch polysaccharides modulate bacterial microbiota, pathways for butyrate production, and abundance of pathogenic Escherichia coli in the pig gastrointestinal tract. Appl Environ Microbiol 76, 3692-3701.

28. Malkki Y, Autio K, Hanninen O, et al. (1992) Oat bran concentrates: physical properties of $\beta$-glucan and hypocholesterolemic effects in rats. Cereal Chem 69, 647-653.

29. van Laar H, Tamminga S, Williams BA, et al. (2002) Fermentation characteristics of polysaccharide fractions extracted from the cell walls of maize endosperm. J Sci Food Agric 82, 1369-1375.

30. Amrein TM, Granicher P, Arrigoni E, et al. (2003) In vitro digestibility and colonic fermentability of aleurone isolated from wheat bran. Lebenson Wiss Technol 36, 451-460.

31. Sayar S, Jannink JL \& White PJ (2007) Digestion residues of typical and high- $\beta$-glucan oat flours provide substrates for in vitro fermentation. J Agric Food Chem 55, 5306-5311.

32. Williams BA, Mikkelsen D, le Paih L, et al. (2011) In vitro fermentation kinetics and end-products of cereal arabinoxylans and $(1,3 ; 1,4)-\beta$-glucans by porcine faeces. $J$ Cereal Sci 53, 53-58

33. Zhao J \& Cheung PKC (2011) Fermentation of $\beta$-glucans derived from different sources by bifidobacteria: evaluation of their bifidogenic effect. J Agric Food Chem 59, 5986-5992.

34. Lin B, Gong J, Wang Q, et al. (2011) In vitro assessment of the effects of dietary fibres on microbial fermentation and communities from large intestinal digesta of pigs. Food Hydrocolloid 25, 180-188.

35. Louis P, Scott KP, Duncan SH, et al. (2007) Understanding the effects of diet on bacterial metabolism in the large intestine. J Appl Microbiol 102, 1197-1208.

36. Vardakou M, Nueno Palop C, Gasson M, et al. (2007) In vitro three-stage continuous fermentation of wheat arabinoxylan fractions and induction of hydrolase activity by the gut microflora. Int J Biol Macromol 41, 584-589.

37. Englyst KN, Liu S \& Englyst HN (2007) Nutritional characterization and measurement of dietary carbohydrates. Eur J Clin Nutr 61, S19-S39.

38. Van Loo J, Coussement P, Leenheer LD, et al. (1995) On the presence of inulin and oligofructose as natural ingredients in the western diet. Crit Rev Food Sci Nutr 35, 525-552.

39. Martinez-Villaluenga C, Frias J \& Vidal-Valverde C (2008) Alpha-galactosides: antinutritional factors or functional ingredients? Crit Rev Food Sci Nutr 48, 301-316.

40. Verspreet J, Pollet A, Cuyvers S, et al. (2012) A simple and accurate method for determining wheat grain fructan content and average degree of polymerization. J Agric Food Chem 60, 2102-2107.

41. Bancal P, Henson CA, Gaudillere JP, et al. (1991) Fructan chemical structure and sensitivity to an exohydrolase. Carbohydr Res 217, 137-151.

42. Bosscher D, Van Loo J \& Franck A (2006) Inulin and oligofructose as prebiotics in the prevention of intestinal infections and diseases. Nutr Res Rev 19, 216-226.

43. Meyer D \& Stasse-Wolthuis M (2009) The bifidogenic effect of inulin and oligofructose and its consequences for gut health. Eur J Clin Nutr 63, 1277-1289.

44. Cani PD, Amar J, Iglesias MA, et al. (2007) Metabolic endotoxemia initiates obesity and insulin resistance. Diabetes 56, 1761-1772

45. Liu T, Hougen H, Vollmer AC, et al. (2012) Gut bacteria profiles of Mus musculus at the phylum and family levels are influenced by saturation of dietary fatty acids. Anaerobe 18, 331-337.

46. de Wit N, Derrien M, Bosch-Vermeulen H, et al. (2012) Saturated fat stimulates obesity and hepatic steatosis and affects gut microbiota composition by an enhanced overflow of dietary fat to the distal intestine. Am J Physiol Gastrointest Liver Physiol 303, G589-G599.

47. Martinez I, Wallace G, Zhang CM, et al. (2009) Diet-induced metabolic improvements in a hamster model of hypercholesterolemia are strongly linked to alterations of the gut microbiota. Appl Environ Microbiol 75, 4175-4184.

48. Tamura M, Hori S, Hoshi C, et al. (2012) Effects of rice bran oil on the intestinal microbiota and metabolism of isoflavones in adult mice. Int J Mol Sci 13, 10336-10349.

49. Martínez I, Perdicaro DJ, Brown AW, et al. (2013) Dietinduced alterations of host cholesterol metabolism are likely to affect the gut microbiota composition in hamsters. Appl Environ Microbiol 79, 516-524.

50. Spencer MD, Hamp TJ, Reid RW, et al. (2011) Association between composition of the human gastrointestinal microbiome and development of fatty liver with choline deficiency. Gastroenterology 140, 976-986.

51. Piironen V, Lindsay DG, Miettinen TA, et al. (2000) Plant sterols: biosynthesis, biological function and their importance to human nutrition. J Sci Food Agric 80, 939-966.

52. Zhao M \& Moghadasian MH (2008) Chemistry, natural sources, dietary intake and pharmacokinetic properties of ferulic acid: a review. Food Chem 109, 691-702.

53. Anson NM, Selinheimo E, Havenaar R, et al. (2009) Bioprocessing of wheat bran improves in vitro bioaccessibility 
and colonic metabolism of phenolic compounds. I Agric Food Chem 57, 6148-6155.

54. Braune A, Bunzel M, Yonekura R, et al. (2009) Conversion of dehydrodiferulic acids by human intestinal microbiota. J Agric Food Chem 57, 3356-3362.

55. Russell WR, Scobbie L, Chesson A, et al. (2008) Anti-inflammatory implications of the microbial transformation of dietary phenolic compounds. Nutr Cancer 60, 636-642.

56. Vitaglione P, Napolitano A \& Fogliano V (2008) Cereal dietary fibre: a natural functional ingredient to deliver phenolic compounds into the gut. Trends Food Sci Technol 19 451-463.

57. Damiani CR, Benetton CA, Stoffel C, et al. (2007) Oxidative stress and metabolism in animal model of colitis induced by dextran sulfate sodium. J Gastroenterol Hepatol 22, 1846-1851.

58. Naito Y, Takagi T \& Yoshikawa T (2007) Neutrophil-dependent oxidative stress in ulcerative colitis. J Clin Biochem Nutr 41, 18-26.

59. Dong WG, Liu SP, Yu BP, et al. (2003) Ameliorative effects of sodium ferulate on experimental colitis and their mechanisms in rats. World J Gastroenterol 9, 2533-2538.

60. Wolever TMS, Spadafora P, Eshuis H, et al. (1991) Interaction between colonic acetate and propionate in humans. Am J Clin Nutr 53, 681-687.

61. Karppinen S, Liukkonen $\mathrm{K}$, Aura AM, et al. (2000) In vitro fermentation of polysaccharides of rye, wheat and oat brans and inulin by human faecal bacteria. J Sci Food Agric 80, 1469-1476.

62. Connolly ML, Lovegrove JA \& Tuohy KM (2010) In vitro evaluation of the microbiota modulation abilities of different sized whole oat grain flakes. Anaerobe 16, 483-488.

63. Kim HJ \& White PJ (2009) In vitro fermentation of oat flours from typical and high $\beta$-glucan oat lines. J Agric Food Chem 57, 7529-7536.
64. Fassler C, Arrigoni E, Venema K, et al. (2006) In vitro fermentability of differently digested resistant starch preparations. Mol Nutr Food Res 50, 1220-1228.

65. Regand A, Chowdhury Z, Tosh SM, et al. (2011) The molecular weight, solubility and viscosity of oat $\beta$-glucan affect human glycemic response by modifying starch digestibility. Food Chem 129, 297-304.

66. Walter J, Martínez I \& Rose DJ (2013) Holobiont nutrition considering the role of the gastrointestinal microbiota in the health benefits of whole grains. Gut Microbes 4, 1-7.

67. Reilly P, Sweeney T, Smith AG, et al. (2010) The effects of cereal-derived $\beta$-glucans and enzyme supplementation on intestinal microbiota, nutrient digestibility and mineral metabolism in pigs. Livest Sci 133, 144-147.

68. Snart J, Bibiloni R, Grayson T, et al. (2006) Supplementation of the diet with high-viscosity $\beta$-glucan results in enrichment for lactobacilli in the rat cecum. Appl Environ Microbiol 72, 1925-1931.

69. Christiansen KL, Weller CL, Schlegel VL, et al. (2007) Extraction and characterization of lipids from the kernels, leaves, and stalks of nine grain sorghum parent lines. Cereal Chem 84, 463-470.

70. Mandak E \& Nyström L (2012) The effect of in vitro digestion on steryl ferulates from rice (Oryza sativa L.) and other grains. J Agric Food Chem 60, 6123-6130.

71. Dall'Asta M, Calani L, Tedeschi M, et al. (2012) Identification of microbial metabolites derived from in vitro fecal fermentation of different polyphenolic food sources. Nutrition $\mathbf{2 8}$, 197-203.

72. Meydani M (2009) Potential health benefits of avenanthramides of oats. Nutr Rev 67, 731-735.

73. Macfarlane GT \& Macfarlane S (2012) Bacteria, colonic fermentation, and gastrointestinal health. J AOAC Int 95, $50-60$. 